\section{(C) OPEN ACCESS}

\title{
Cancer incidence in female laboratory employees: extended follow-up of a Swedish cohort study
}

\author{
Per Gustavsson, ${ }^{1,2}$ Tomas Andersson, ${ }^{2}$ Annika Gustavsson, ${ }_{1}^{2}$ Christina Reuterwall ${ }^{3}$
}

${ }^{1}$ Unit of Occupational Medicine, Institute of Environmental Medicine, Karolinska Institutet, Stockholm, Solnavägen 4, Sweden

${ }^{2}$ Centre for Occupational and Environmental Medicine, Stockholm County Council, Stockholm, Solnavägen 4, Sweden

${ }^{3}$ Unit of Research, Education and Development, Östersund Hospital, Östersund, Sweden

\section{Correspondence to}

Professor Per Gustavsson, Unit of Occupational Medicine,

Karolinska Institutet, Solnavägen 4, 113 65, Stockholm, Sweden; per. gustavsson@ki.se

Received 3 November 2016 Revised 29 March 2017 Accepted 9 April 2017 Published Online First 8 June 2017

\section{CrossMark}

To cite: Gustavsson $\mathrm{P}$ Andersson T, Gustavsson A, et al. Occup Environ Med 2017;74:823-826.

\section{ABSTRACT}

Objectives Work in chemical laboratories is associated with exposure to chemicals, of which some are known or suspected carcinogens. A cohort study of laboratory workers in Stockholm followed until 1992 showed an excess of hematolymphatic malignancies in chemical laboratories and an excess of breast cancer among women working for more than 10 years in such laboratories. The follow-up of this cohort has now been extended by 20 years.

Methods The cohort comprised 2245 female laboratory workers who are employed for $>1$ year from 1950 to 1989. Information on employment periods and type of laboratory ('chemical' or 'non-chemical') was obtained from employee registers. Cancer diagnoses from 1958 to 2012 were obtained from the Swedish Cancer Registry.

Results There were 383 cases of cancer ( $\mathrm{SIR}=0.93$ (95\% Cl 0.84 to 1.02$))$. The risk of breast cancer was elevated, of borderline statistical significance, among those who had worked for at least 10 years in chemical laboratories ( $\mathrm{SIR}=1.41$ (95\% CI 0.99 to 1.95$) 36$ cases). The breast cancer risk was especially high in women who had worked for more than 10 years in chemical labs before 1970 (SIR=3.76 (95\% Cl 1.72 to 7.14$)$, nine cases). There was no excess of breast cancer in non-chemical labs (SIR=0.77 (95\% CI 0.54 to 1.07), 35 cases). The number of hematolymphatic cancer was no longer significantly elevated.

Conclusions The increased risk of breast cancer, as well as the earlier noted excess of hematolymphatic malignancies, may be related to exposure to carcinogenic chemicals/organic solvents (eg, benzene) used in chemical laboratories, especially during earlier periods.

\section{BACKGROUND}

Work in chemical laboratories may involve exposure to a large number of chemicals, of which some are known or suspected carcinogens. ${ }^{1}$ Studies of male and female US chemists indicated an excess of deaths from malignant lymphomas and pancreatic cancer, as well as from cancer of the breast and hematolymphatic organs, respectively. ${ }^{2} 3$ Excesses of hematolymphatic malignancies were found both among British ${ }^{4}$ and Swedish ${ }^{5}$ chemists. A cluster of sarcomas and lymphomas at Institute Pasteur in France prompted a series of investigations reviewed by Rachet et al ${ }^{1}$ concluding that more consistently reported were cancer of the pancreas, brain and non-Hodgkin's lymphoma. A study of Finnish laboratory workers showed no excess of cancer, although the follow-up was short. ${ }^{6}$ An investigation of US chemical research laboratory workers

\section{What this paper adds}

- Previous studies of chemical lab workers and chemists show an excess of various forms of cancer, specifically of the hematolymphatic organs and breast.

- The study reports the findings from an extension of follow-up by 20 years in a cohort of female laboratory workers in Stockholm.

- The study showed an increased risk of breast cancer among women in chemical labs, especially pronounced in, but not delimited to, those who had worked for more than 10 years before 1970.

- An excess of hematolymphatic malignancies found in an earlier follow-up of this cohort was not confirmed during the prolonged follow-up.

- Further action to assure safe working conditions in laboratories is warranted.

showed increased mutagenicity in urine compared with non-lab workers. ${ }^{7}$

Cancer of the breast has been reported in excess in some but not all studies of female laboratory workers or in occupations involving exposure to organic solvents. ${ }^{3-11}$ It has been hypothesised that the lipophilic properties and accumulation in breast tissue of potentially carcinogenic solvents may play a role in the process. ${ }^{8}$

We have earlier reported on the cancer incidence from 1958 to 1992 in a cohort of laboratory workers in Stockholm, Sweden. An increased risk of hematolymphatic tumours was found among women who had worked in chemical laboratories, and an excess of breast cancer was found in women who had worked for more than 10 years in such laboratories. ${ }^{9}$ We here report an extended follow-up of the women in this cohort.

\section{MATERIAL AND METHODS}

The methods have been described in detail earlier. ${ }^{9}$ Briefly, data on civil registration number, name, job titles and type of laboratory were collected from employment records at Karolinska Institutet and Karolinska Hospital in Stockholm. Laboratory workers employed for at least 1 year between 1950 and 1989 were included. Due to changes in the employee record keeping system, it was not feasible to extend data on employment periods after 1989 , and the present analysis was based on employment records up to 1989 . Of the 2245 women in the cohort, $1493(67 \%)$ had terminated their 
employment before 1989, leaving 752 women (33\%) for which the employment duration may have been underestimated.

Based on assessments by an occupational hygienist, laboratories were classified as 'chemical' (chemical analytical routine and research laboratories, pathology, cytology and genetic laboratories) or 'non-chemical' (microbiology, physiology, endocrinology, haematology and fertility). The subcohort 'chemical lab workers' $(n=1246)$ included those who had worked for at least 1 year in labs classified as chemical, but they may in addition have worked in labs classified as 'non-chemical'. The subcohort of 'never chemical lab workers' $(n=666)$ had never worked in chemical labs (even for a short time). Workers who had worked in chemical labs for less than a year but fulfilled the entry criterion of $\geq 1$ year of work in any lab did not qualify for inclusion neither among 'chemical labs workers' nor among 'never worked in chemical labs' $(n=333)$ but were retained in the full cohort. The calculation of employment duration was made specific for type of lab, that is, workers in the group ' $>10$ years in chemical labs', includes only those who had worked at least 10 years in such labs.

The cohort was followed for cancer incidence in the national Swedish Cancer Registry from 1958 up to 2012. The present matching included also the earlier reported follow-up period (1958-1992). The registry has, since it started in 1958, classified cancer sites both according to International Classification of Diseases (ICD), Revision 7 and the ICD revision in use for successive years. Cancer sites are here reported according to ICD-7. Date of death, date of emigration and vital status at end of follow-up were obtained from Statistics Sweden. The SIR was estimated by the person-year method, stratifying by 5-year classes of age and calendar time, using the general female population in Stockholm County as reference. Persons were under risk of cancer from beginning of study (1 January 1958) or 1 year after start of work in the type of lab studied, whichever occurred last, and until end of study (31 December 2012), death or emigration, whichever occurred first. Those lost to follow-up were considered under risk until end of employment. The analyses were performed with SAS software V.9.4.

The study was approved by the Regional Ethics Committee in Stockholm (2014/233-31/4).

\section{RESULTS}

The original cohort comprised 2247 female and 306 male laboratory workers. This update focused on the women in the cohort. Of these, two women (non-cases) were found not to fulfil the entry criterion of 1 year of employment and were excluded from the study. The remaining 2245 women were included and contributed 79943 person-years of observation. For the entire study period from 1958 to 2012, there were 383 cancer tumours, 260 women had died, 171 had emigrated and 6 were lost to follow-up. During the extended follow-up from 1993 to 2012, 296 new cancers were identified. The new matching for the period 1958-1992 identified three cases less than what was found in the matching done in 1992. This is likely to be a result of corrections made by the registry, and we here present findings from the most recent matching of the cohort.

The mean age of the cohort members at beginning of follow-up was 28.6 years (SD 8.4), 46.4 years (SD 11.0) at end of follow-up in 1992 and 63.8 years (SD 12.1) at end of follow-up in 2012. Table 1 presents the risk of cancer for sites with at least five cases and sites of particular interest. In the full cohort, the overall cancer incidence was slightly lower than expected. The incidence of breast cancer was not elevated in the full cohort, although there was an elevation of borderline statistical significance among women who had worked in chemical labs for $>10$ years (SIR 1.41 (95\% CI 0.99 to 1.95$)$ ) based on 35 cases. No excess of breast cancer was found among those who had never worked in chemical labs, neither for $>1$ year (SIR $0.77(95 \%$ CI 0.54 to 1.07 )) nor for $>10$ years (SIR 0.87 (95\% CI 0.45 to 1.53$)$ ). During the extended follow-up from 1993 to 2012, there were 23 new cases of breast cancer among those who had worked $>10$ years in chemical labs, corresponding to an SIR of 1.18 (95\% CI 0.75 to 1.77$)$. Further investigations of breast cancer risk in relation to time of employment showed that the excess was especially pronounced in women who had accumulated 10 years of work in chemical labs before 1970 (when benzene to a large extent had been phased out from the labs) $(\mathrm{SIR}=3.76$ (95\% CI 1.72 to 7.14$)$ ) based on nine cases. Among women who had $>10$ years of work in chemical labs but started after 1970, the SIR was 1.37 (95\% CI 0.82 to 2.14) based on 19 cases.

An elevated risk of non-melanoma skin cancer (NMSC) that was noted in the full cohort was mainly driven by an excess among those who had never worked in chemical laboratories.

The earlier finding of an increased risk of hematolymphatic malignancies in this cohort was not confirmed during this prolonged follow-up. Among those ever employed in chemical labs, there were seven new cases in the extended follow-up, whereas 9.8 were expected. The SIR among those who had worked for more than 10 years in chemical labs was still elevated for the entire study period of 1958-2012 but not statistically significant: SIR 1.50 (95\% CI 0.60 to 3.09). There were few leukaemias, and a subdivision of lymphatic and myeloid leukaemias was not informative due to low numbers.

A new finding was an elevated risk of cancer of the mouth among those who had worked in non-chemical labs. The risk was more pronounced among those employed for more than 10 years, although with a wide CI. No cases of cancer of the stomach or kidney were observed in the cohort.

\section{DISCUSSION}

A main finding was an elevated risk of breast cancer of borderline statistical significance among long-time employed women working in labs with a high probability of contact with chemicals and organic solvents. The risk was especially pronounced among women who had worked for more than 10 years in such labs before 1970. A second finding was that the excess of malignancies of the hematolymphatic system found in the earlier follow-up was not confirmed in the extended follow-up period.

A review of breast cancer and exposure to organic solvents published in 1997 presented a hypothesis that organic solvents could induce breast cancer by a high accumulation of lipophilic solvents in the fat-rich breast tissue. ${ }^{8}$ A later published record linkage study from the Nordic countries consistently showed statistically increased risks of breast cancer in laboratory assistants in Denmark, Finland, Norway as well as in Sweden. ${ }^{10}$ A population-based study in Poland showed an increased risk of hormone-receptor negative breast cancer among women exposed to organic solvents. ${ }^{11}$ Our findings add to the evidence that occupational exposure to chemicals and organic solvents may induce breast cancer in women.

Work in chemical laboratories may be associated with exposure to a large number of chemical substances, and exposure conditions may vary from lab to lab and over time. A potential explanation for the excess risk of breast cancer in this cohort is exposure to the carcinogenic solvent benzene, 
Table 1 Cancer risk from 1958 to 2012 in 2245 female laboratory workers in Stockholm, Sweden, by type of laboratory and duration of work

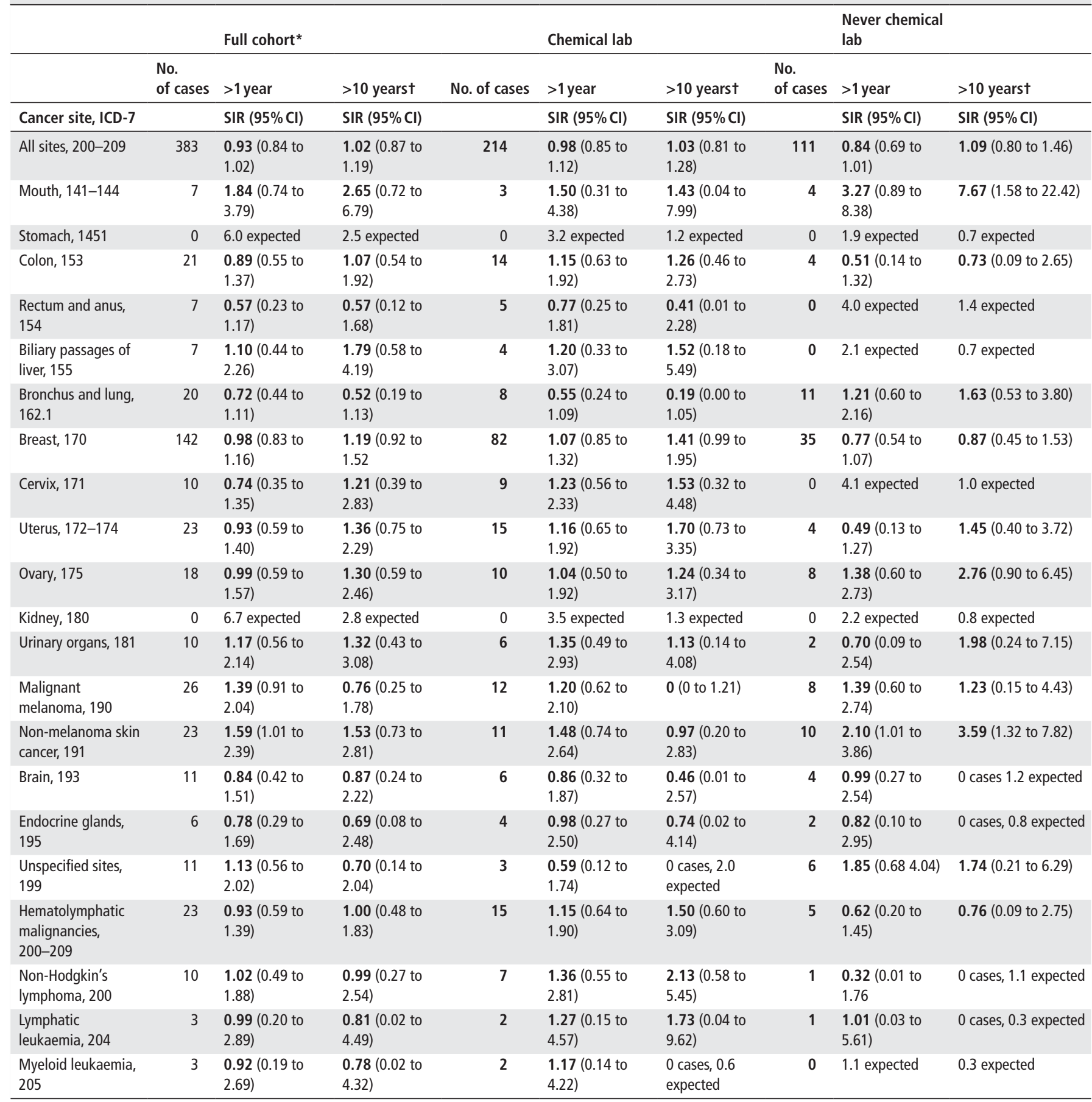

*The sum of persons in the subcohorts of 'chemical' and 'never chemical' lab workers is less than the number in the full cohort. See text in methods section for explanation. tTen years of work up to and including 1989.

ICD-7, International Classification of Diseases, Revision 7.

earlier widely used in chemical labs, and an established cause of hematolymphatic malignancies. Benzene was phased out during the 1960s in Sweden, ${ }^{9}$ but several of the women in this cohort may have been exposed to benzene in the beginning of their occupational career. An analysis of the risk of breast cancer in relation to time period of work showed that the breast cancer risk was especially pronounced among those who had attained more than 10 years of work in chemical labs before 1970 . The risk was elevated, but not statistically significant, in those who had started work in chemical labs after 1970. Work in chemical labs may still involve exposure to a number of other carcinogenic compounds, for example chlorinated hydrocarbons. ${ }^{12}$ The phasing out of benzene may also explain why no excess risk for hematolymphatic malignancies was seen during this extended follow-up period, in contrast to the earlier follow-up.

Breast cancer risk is strongly related to hormonal factors associated with reproduction. It is not likely, though, that these factors could differ substantially between workers in chemical and non-chemical labs. Work at night has been proposed as a risk factor for breast cancer, but work at night was not common among the laboratory employees in this cohort. 
This study have strengths in its relatively large size, detailed work histories obtained from an objective source (employment records), the almost complete follow-up and the high accuracy of the Swedish Cancer Registry. This study's weakness is that work histories were not updated after 1989, which concerned 33\% of the cohort. Of these, some workers may have acquired more than 10 years of work and are missing in the category of $>10$ years in this follow-up. However, there is no 'dilution' with less exposed persons in the $>10$ years category, and in addition, those acquiring more than 10 years of work during later years probably had a lower exposure, which would reduce the misclassification from this lack of information.

An excess of NMSC was found specifically among those working in non-chemical labs. We have no explanation for this, but it should be noted that there was an excess of malignant melanoma among male (but not female) laboratory assistants in the Nordic countries. ${ }^{10}$ Exposure to sunlight and UV-irradiation are risk factors both for NMSC and malignant melanoma, but such exposure is not typical for the non-chemical labs. The excess deserves attention in future studies of non-chemical lab workers, and so does the excess of cancer of the mouth found in the non-chemical lab workers. We have no explanation for the deficit of cancers of the kidney and stomach, which may be chance findings.

Acknowledgements Authors are grateful to Gloria Manrique, Anna Boberg and Cecilia Rudengren for their assistance in collection of cohort data from employee registers.

Contributors $P G$, the principal investigator of the study, and CR conceived and designed the study. TA and AG performed data matching and statistical analysis. All authors contributed to the interpretation of the results and revised and approved the manuscript.

Funding The study was financed by a grant from the Swedish Research Council for Health, Working Life and Welfare (FORTE), grant no. 2013-0402.

Competing interests None declared.
Ethics approval Regional Ethics Committee in Stockholm (2014/233-31/4).

Provenance and peer review Not commissioned; externally peer reviewed.

Open Access This is an Open Access article distributed in accordance with the Creative Commons Attribution Non Commercial (CC BY-NC 4.0) license, which permits others to distribute, remix, adapt, build upon this work non-commercially, and license their derivative works on different terms, provided the original work is properly cited and the use is non-commercial. See: http://creativecommons.org/ licenses/by-nc/4.0/

(c) Article author(s) (or their employer(s) unless otherwise stated in the text of the article) 2017. All rights reserved. No commercial use is permitted unless otherwise expressly granted.

\section{REFERENCES}

1 Rachet B, Partanen T, Kauppinen T, et al. Cancer risk in laboratory workers: an emphasis on biological research. Am J Ind Med 2000;38:651-65.

2 Li FP, Fraumeni JF, Mantel N, et al. Cancer mortality among chemists. J Nat/ Cancer Inst 1969:43:1159-64.

3 Walrath J, Li FP, Hoar SK, et al. Causes of death among female chemists. Am J Public Health 1985;75:883-5.

4 Hunter WJ, Henman BA, Bartlett DM, et al. Mortality of professional chemists in England and Wales, 1965-1989. Am J Ind Med 1993;23:615-27.

5 Olin GR, Ahlbom A. The Cancer mortality among swedish chemists graduated during three decades. A comparison with the general population and with a cohort of architects. Environ Res 1980;22:154-61.

6 Kauppinen T, Pukkala E, Saalo A, et al. Exposure to chemical carcinogens and risk of cancer among finnish laboratory workers. Am J Ind Med 2003;44:343-50.

7 Varella SD, Rampazo RA, Varanda EA. Urinary mutagenicity in chemical laboratory workers exposed to solvents. J Occup Health 2008;50:415-22.

8 Labrèche FP, Goldberg MS. Exposure to organic solvents and breast cancer in women: a hypothesis. Am J Ind Med 1997:32:1-14.

9 Gustavsson P, Reuterwall C, Sadigh J, et al. Mortality and cancer incidence among laboratory technicians in medical research and routine laboratories (Sweden). Cancer Causes Control 1999;10:59-64.

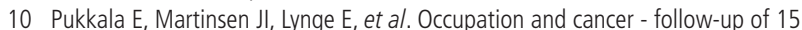
million people in five nordic countries. Acta Oncol 2009;48:646-790.

11 Peplonska B, Stewart P, Szeszenia-Dabrowska N, et al. Occupational exposure to organic solvents and breast cancer in women. Occup Environ Med 2010;67:722-9.

12 Lynge E, Anttila A, Hemminki K. Solvents Organic and cancer. Cancer Causes and Control 1997;8:406-19. 